\title{
Gastric Cancer Cell Lines Have Different MYC-Regulated Expression Patterns but Share a Common Core of Altered Genes
}

\author{
Jersey Heitor da S. Maués $\left(\mathbb{D},{ }^{1}\right.$ Helem Ferreira Ribeiro $\mathbb{D D}^{1,2}$ Giovanny R. Pinto, ${ }^{3}$ \\ Luana de Oliveira Lopes, ${ }^{3}$ Letícia M. Lamarão, ${ }^{4}$ Carla Mariana F. Pessoa, ${ }^{5}$ \\ Caroline de Fátima Aquino Moreira-Nunes, ${ }^{6}$ Raimundo Miranda de Carvalho, ${ }^{7}$ \\ Paulo P. Assumpção, ${ }^{5}$ Juan A. Rey, ${ }^{8}$ and Rommel M. Rodríguez Burbano ${ }^{7}$ \\ ${ }^{1}$ Human Cytogenetics Laboratory, Institute of Biological Sciences, Federal University of Pará, Belém, Brazil \\ ${ }^{2}$ Center of Biological and Health Sciences, Department of Biomedicine, University of Amazon, Belém, Brazil \\ ${ }^{3}$ Department of Biomedicine, Federal University of Piauí, Parnaíba, Brazil \\ ${ }^{4}$ Laboratory of Nucleic Acids, State Center of Hematology and Hemotherapy, Belém, Brazil \\ ${ }^{5}$ Oncology Research Nucleus, University Hospital João de Barros Barreto, Federal University of Pará, Belém, Brazil \\ ${ }^{6}$ Laboratory of Pharmacogenetics, Drug Research and Development Center, Federal University of Ceará, Fortaleza, Brazil \\ ${ }^{7}$ Laboratory of Molecular Biology, Ophir Loyola Hospital, Belém, Brazil \\ ${ }^{8}$ Molecular Oncogenetics Laboratory, Research Unit, Hospital Universitario La Paz, Madrid, Spain
}

Correspondence should be addressed to Jersey Heitor da S. Maués; jerseymaues@gmail.com

Received 27 April 2018; Revised 12 September 2018; Accepted 23 September 2018; Published 16 October 2018

Guest Editor: Kiran L. Sharma

Copyright (C) 2018 Jersey Heitor da S. Maués et al. This is an open access article distributed under the Creative Commons Attribution License, which permits unrestricted use, distribution, and reproduction in any medium, provided the original work is properly cited.

MYC is an oncogene responsible for excessive cell growth in cancer, enabling transcriptional activation of genes involved in cell cycle regulation, metabolism, and apoptosis, and is usually overexpressed in gastric cancer (GC). By using siRNA and Next-Generation Sequencing (NGS), we identified MYC-regulated differentially expressed Genes (DEGs) in three Brazilian gastric cancer cell lines representing the histological subtypes of GC (diffuse, intestinal, and metastasis). The DEGs were picked using Sailfish software, followed by Gene Set Enrichment Analysis (GSEA) and Kyoto Encyclopedia of Gene and Genome (KEGG) pathway analysis using KEGG. We found 11 significantly enriched gene sets by using enrichment score (ES), False Discovery Rate (FDR), and nominal P-values. We identified a total of $5.471 \mathrm{DEGs}$ with correlation over (80\%). In diffuse-type and in metastatic GC cell lines, MYCsilencing caused DEGs downregulation, while the intestinal-type GC cells presented overall DEGs upregulation after MYC siRNA depletion. We were able to detect 11 significant gene sets when comparing our samples to the hallmark collection of gene expression, enriched mostly for the following hallmarks: proliferation, pathway, signaling, metabolic, and DNA damage response. When we analyzed our DEGs considering KEGG metabolic pathways, we found 12 common branches covering a wide range of biological functions, and three of them were common to all three cell lines: ubiquitin-mediated proteolysis, ribosomes, and system and epithelial cell signaling in Helicobacter pylori infection. The GC cell lines used in this study share 14 MYC-regulated genes, but their gene expression profile is different for each histological subtype of GC. Our results present a computational analysis of $M Y C$-related signatures in GC, and we present evidence that GC cell lines representing distinct histological subtypes of this disease have different MYC-regulated expression profiles but share a common core of altered genes. This is an important step towards the understanding of MYC's role in gastric carcinogenesis and an indication of probable new drug targets in stomach cancer.

\section{Introduction}

Gastric cancer (GC) remains as an important cause of cancerrelated morbidity and mortality worldwide, with recent estimates accounting for over 950.000 new diagnosis and 720.000 deaths each year [1]. Treatment of GC at advanced stages remains difficult, and the prognosis is still poor, partly as a result of local recurrence, tumor invasion, and/or metastasis [2]

The MYC oncogene, located at 8q24, is a key oncogene in gastric carcinogenesis, and an increase in both copy number 
TABLE 1: Samples used in this study. Control siRNA samples are labeled as C and MYC-siRNA as M.

\begin{tabular}{lccc}
\hline Cell line & Samples (GEO) & Sample Name & GC Histological subtype \\
\hline \multirow{2}{*}{ AGP01 } & GSM2147866 & $1 \mathrm{C}$ & Ascitic fluid of intestinal GC \\
& GSM2147867 & $1 \mathrm{M}$ & Ascitic fluid of intestinal GC \\
ACP02 & GSM2147868 & $2 \mathrm{C}$ & Diffuse \\
& GSM2147869 & $2 \mathrm{M}$ & Diffuse \\
ACP03 & GSM2147870 & $3 \mathrm{C}$ & Intestinal \\
& GSM2147871 & $3 \mathrm{M}$ & Intestinal \\
\hline
\end{tabular}

and mRNA expression was classified as one of the driver mutations in gastric tumors [3]. MYC amplification and overexpression are present in $6-58 \%$ of all sporadic gastric tumors [4-6], being more frequent in Brazilian samples [79], usually as a result from gene amplification and chromosomal translocations $[2,10]$.

Our research group previously reported that MYC mRNA and protein overexpression is a common finding in GC samples and in some preneoplastic gastric lesions [7, 11-14] from a Brazilian population, as well as in nonhuman primate models of gastric carcinogenesis [15]. We also established and characterized three GC cell lines, AGP01, ACP02, and ACP03, obtained from intestinal-type GC metastasis, diffuse-type GC, and intestinal-type GC, respectively (Leal et al. 2009). Those cell lines also carry genetic alterations commonly found in Brazilian GC patients, such as MYC amplification and overexpression and TP53 deletion $[7,13,16]$.

Some consequences of excessive intracellular MYC levels are genomic instability [17] and error-prone DNA replication caused by oncogene-induced replicative stress [18]. Even though there is an association between an increase in $M Y C$ expression and gastric cancer, its exact role in gastric tumorigenesis is not yet fully understood $[19,20]$ and most of the high-throughput studies carried so far concerning gastric cancer genetics overlook MYC's importance in this process $[2,3,21-24]$

Bioinformatics has mostly been applied in basic science research. Following the completion of human genome sequencing, it has also facilitated numerous discoveries in basic medicine, and several clinical applications of bioinformatics have been reported, including clinical sequencing, an emerging field of precision medicine [25]. In cancer research, bioinformatics has been used to study cancer transcriptome, early diagnosis, cancer grading, and prognosis prediction [26].

In this study, we used RNA interference (RNAi) to block MYC's mRNA translation, followed by Ion Proton ${ }^{\mathrm{TM}}$ semiconductor sequencing, in order to identify MYC's regulation signature in AGP01, ACP02, and ACP03 cell lines. We found 11 common pathways for the GC cell lines, which we believe can help in the understanding of expression signatures in different GC histological subtypes.

\section{Materials and Methods}

2.1. Cell Lines and siRNA Transfection. Three GC cell lines previously established and characterized by our group were used: AGP01, ACP02, and ACP03 [27]. The three cell lines present chromosome 8 trisomy, MYC amplification [13, 27], and TP53 deletion, which are common genetic alterations in Brazilian gastric cancer patients [28] and in another GC cell line developed in Brazil [29]. A cell culture of nonneoplastic gastric mucosa cells (MNP01, Normal Gastric Mucosa Cell Line 01) pooled from 10 patients without gastric cancer or any other gastric disease, was also used to evaluate the gene and protein expression after $M Y C$-silencing and to validate the knockdown results, as well as the $M Y C$-regulated genes identified after NGS.

A total of $3 \times 10^{5}$ cells were seeded into $6 \mathrm{~cm}^{2}$ plates for each cell line for $24 \mathrm{~h}$ before transfection. Small interfering RNAs (siRNA) targeting MYC (ON-TARGETplus Human MYC (4609) siRNA Dharmacon, EUA) or scrambled control siRNAs (ON-TARGETplus Non-Targeting Pool, Dharmacon, EUA) were transfected into AGP01, ACP02, and ACP03 cell lines using Lipofectamine RNAiMAX Transfection Reagent (Thermo Fisher Scientific, EUA). Optimal transfection was reached after $48 \mathrm{~h}$, and total RNA and proteins were extracted with TRIzol reagent (Thermo Fisher Scientific, EUA). All siRNA experiments were performed three times. The sample names and GEO access codes are shown in Table 1. All siRNA experiments were carried out in biological triplicates.

2.2. Semiconductor Sequencing and Data Pretreatment. Total RNA samples were first treated with DNAse-I to remove any possible DNA contamination, and then the mRNA was enriched using Dynabeads Oligo $(\mathrm{dt})_{25}$ (Thermo Fisher Scientific, USA). The enriched mRNA was fragmented in smaller fragments of $200 \mathrm{bps}$ approximately, which were attached to adapters with known sequences that were unique for each sample. Samples were connected to magnetic beads containing complementary sequences for the adapters and then inserted in microwells where an emulsion-PCR for cDNA synthesis was carried (illustra Ready-To-Go RT-PCR Beads, GE Lifesciences). Our six cDNA libraries were submitted to quantification and quality control using Agilent 2100 Bioanalyzer and were then loaded in Ion Proton V2 PI chip using the Ion $\mathrm{PI}^{\mathrm{TM}} 200$ Sequencing Kit v3 and sequenced using Ion Proton $^{\mathrm{TM}}$ (Thermo Fisher Scientific, EUA) platform in a single multiplex run.

Raw data reads obtained by primary sequencing using Ion Proton ${ }^{\mathrm{TM}}$ were submitted to quality control to calculate alignment and to assess how the reads behave when compared to the reference human genome (Hg19/GRCh37). The aligned reads were mapped and quantified using TMAP (Torrent Mapping Alignment Program), which supports different alignment algorithms [30-32]. Processed datasets were 
TABLE 2: Reads quantification after MYC-siRNA.

\begin{tabular}{lcccc}
\hline Sample name & Total Reads & Total Mapped Reads & Gene mapping rate & Expressed genes \\
\hline 1C & 13.319 .736 & 12.944 .452 & $97.18 \%$ & 14.594 \\
$1 \mathrm{M}$ & 11.043 .607 & 10.771 .298 & $97.53 \%$ & 10.709 \\
2C & 12.021 .142 & 11.751 .830 & $97.76 \%$ & 9.859 \\
2M & 11.701 .695 & 11.481 .863 & $98.12 \%$ & 8.793 \\
3C & 12.772 .535 & 12.546 .479 & $98.23 \%$ & 7.988 \\
3M & 12.430 .807 & 12.112 .157 & $97.44 \%$ & 10.685 \\
\hline
\end{tabular}

uploaded to GEO (Gene Expression Omnibus) under the access number GSE81265.

2.3. Identification and Statistical Analysis of DEGs. Sailfish software pack [33] and the RPKM (Reads Per Kilobase per million mapped reads) [34, 35] were used to significance analysis of DEGs between control and MYC-silenced samples. To identify the DEGs between two paired samples, we used the Audic-Claverie test [36]. Fold-change (FC) was calculated as the $\log _{2}$ ratio between the silenced (M) and the control (C) sample. We used a p-value correction corresponding to differential expression tests using Bonferroni correction [37]. Our cut-off for DEGs definition was established as a False Discovery Rate $($ FDR $)<0.05$ [38] and $|\log 2(\mathrm{FC})|>1$. DEGs were plotted using Multiplot v2, which exhibits personalized gene expression profiles (http://software.broadinstitute.org/cancer/software/genepattern/modules/docs/multiplot/2).

2.4. Functional Enrichment Analysis. We used Gene Set Enrichment Analysis (GSEA) [39] to identify significantly enriched gene sets between siRNA control versus MYCsilenced and vice versa. The gene expression changes perceived by DEGs were related to biologically enriched pathways found in GSEA. The gene expression datasets used were collections $\mathrm{H}$ (Hallmark gene set) [40] and C2 (curated gene set: KEGG), publicly available at MsigDB [41]. The standard parameters defined by Subramanian et al. were used in our analysis. The statistical significance of GSEA analysis was determined by 100 permutations, the enrichment maps were created to significant $(P<0.05$ and False Discovery Rate $($ FDR $)<0.25)$ gene sets, and GraphPad Prism ${ }^{\mathrm{TM}}$ Software was used to graphically represent our data.

2.5. Real-Time Quantitative PCR (RT-qPCR). To confirm the silencing effect of siRNA on $M Y C$ expression, we used real-time quantitative PCR to evaluate its expression in relation to the expression found for the normal gastric mucosa cell line MN01. All tests were made in triplicate and using TaqMan ${ }^{\circledR}$ probes as assays-on-demand products for gene expression (Life Technologies, EUA) (MYC: Hs00153408_ml). The fourteen DEGs identified by our analysis (SKIV2L2: Hs00299011_ml, SRPRB: Hs00253639_ml, JUNB: Hs00357891_s1, BNIP3: Hs00969291_ml, RAB22A: Hs00221082_ml, TMED2: Hs00607277_ml, ACAT1: Hs00608002_ m1, NDUFV2: Hs00221478_m1, LBR: Hs01032700_m1, NCL: Hs01066668_m1, AAAS: Hs00210351_m1, ATXN2:
Hs00268077_ml, LGMN: Hs00271599_ml, and CDKN1B: Hs00153277_ml) were also analyzed and validated by realtime quantitative PCR. The expression of those genes was calculated relative to their expression in the normal gastric mucosa cell line MN01.

\section{Results}

3.1. Quantification of GCs Transcripts and Identification of DEGs Using NGS. In this study, we used next-generation sequencing based in semiconductors, as well as RNA-Seq, to quantify the transcripts and its isoforms in three gastric cancer cell lines, ACP02, ACP03, and AGP01, before and after $M Y C$-silencing using siRNA. The use of siRNA to reduce $M Y C$ expression in the three gastric cancer cell line used in this study was very effective, reducing MYC mRNA expression in $73 \%$ for AGP01, in $84 \%$ for ACP02, and in $77 \%$ for ACP03.

Our NGS sequencing of six libraries generated over 75 million reads, which, after enrichment, were mapped within the reference genome in over $99 \%$ of the samples and in over $98 \%$ of the reference transcriptome (Table 2), and the distribution of the amplified segments was consistent in all samples. Table 2 also shows in average how many genes were identified for each sample. The average reads produced by Ion Proton $^{\mathrm{TM}}$ ranged between 125 and 130 bps.

According to our cut-off (FDR $<0.05$ and $\left|\log _{2}(\mathrm{FC})\right|>$ 1), we obtained a distinct amount of DEGs between siRNA control versus MYC -silenced samples. Using Multiplot (v2), we identified 1.556 downregulated and 917 upregulated DEGs for AGP01; for the diffuse-type cell line (ACP02), we found 4.098 downregulated versus 1.229 upregulated DEGs; finally, for ACP03, an intestinal-type cell line, we identified 3.272 upregulated versus 842 downregulated DEGs (Figure 1). Our results indicate that it is possible to discern histological subtypes of GC by analyzing its MYC-related gene expression pattern.

A total of 16.777 genes from our six datasets obtained by RNA-Seq (GSE81265) were inserted in an expression matrix normalized by RPKM and, after that, used for enrichment comparing with expression datasets from collections $\mathrm{H}$ and C2. By applying GSEA, we looked for gene sets which presented enrichment only in siRNA control samples, but not in $M Y C$-silenced samples, likely MYC targets, and found 11 significant gene sets $(P<0.05$ and FDR $<0.25)$, as shown in Table 3. We found a total of 7903 genes, and 5471 (69.2\%) are enriched in siRNA control samples, presenting a very high correlation between biological replicates and libraries (80\%). 


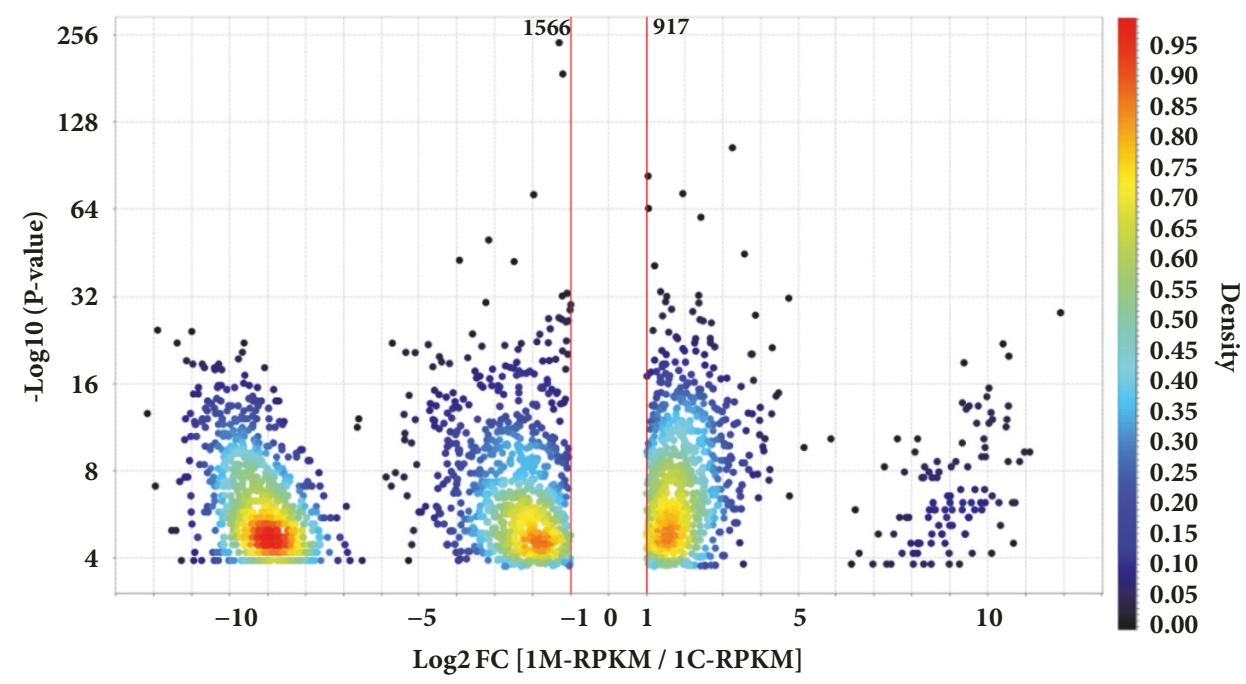

(a)

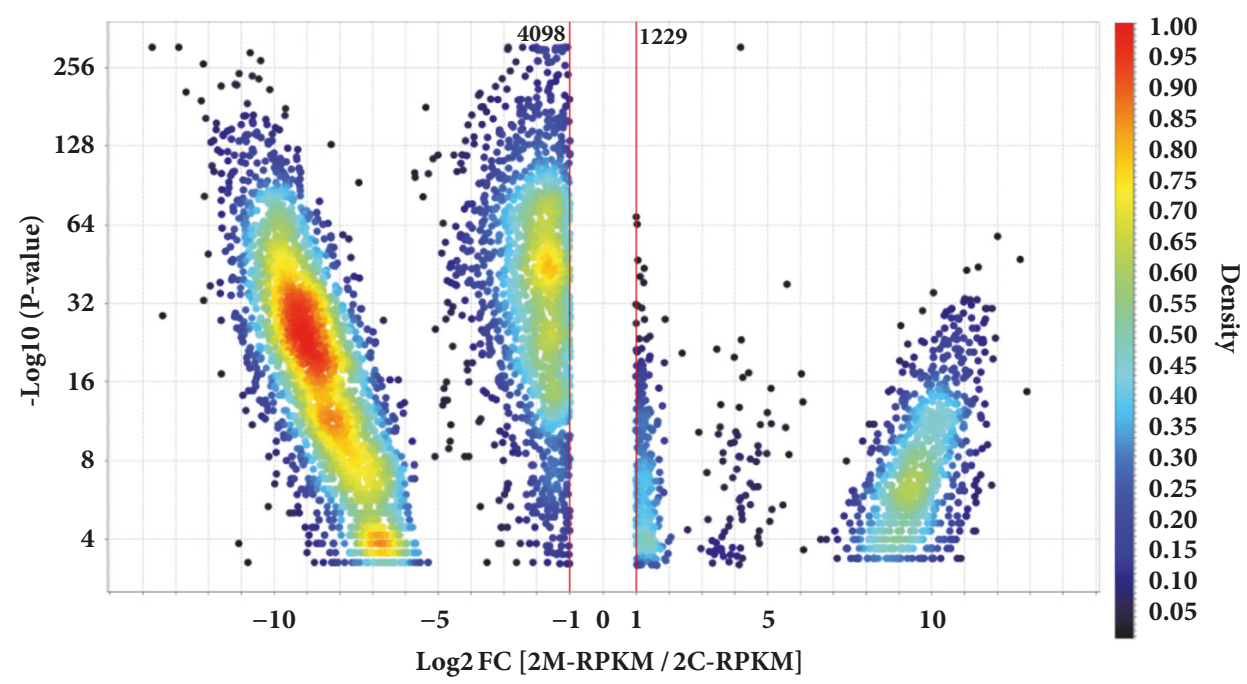

(b)

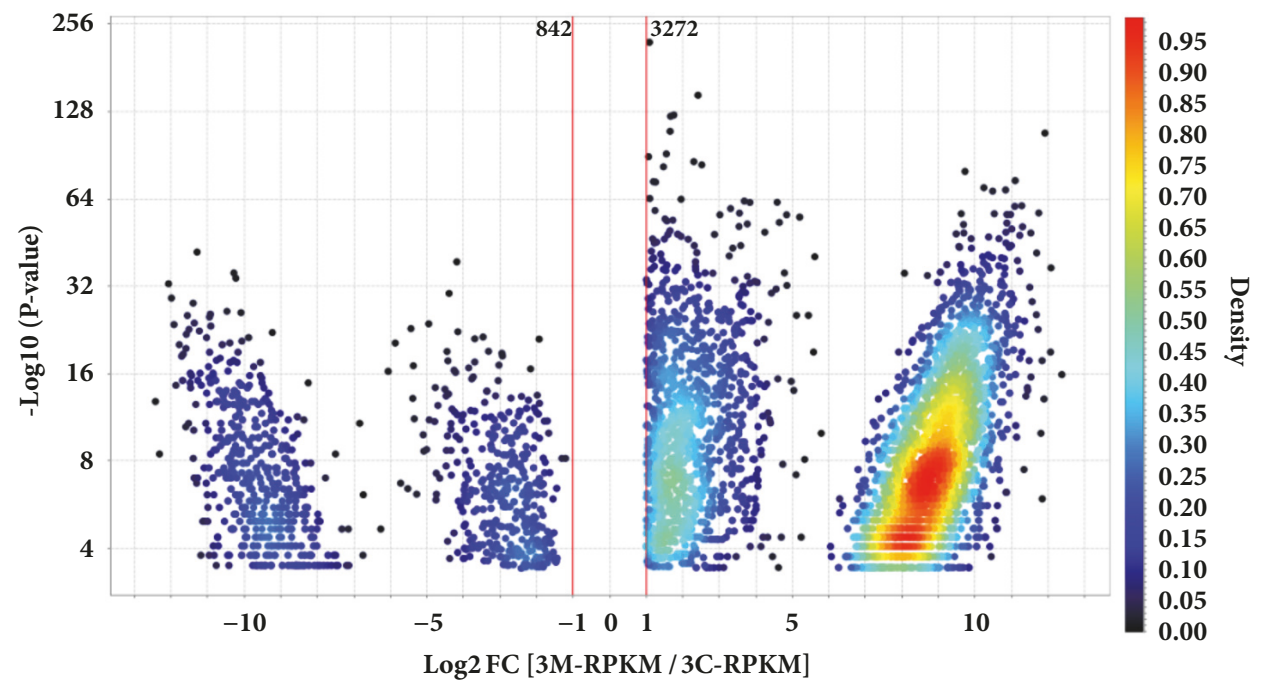

(c)

Figure 1: Volcano plots of DEGs for three GC cell lines after MYC-silencing. $\log _{2}$ Fold-change and P-values (- $\left.\log _{10}\right)$ are shown for DEGs with $\left(\left|\log _{2}(\mathrm{FC})\right|>1\right.$ and $\mathrm{p} \leq$ 0.05). (a) DEGs for AGP01. (b) DEGs for ACP02. (c) DEGs for ACP03. Density is a special-case calculation which is only used in advanced shading. It considers only the operands for this calculation are the $\mathrm{X}$ and $\mathrm{Y}$ values of the graph axes. 


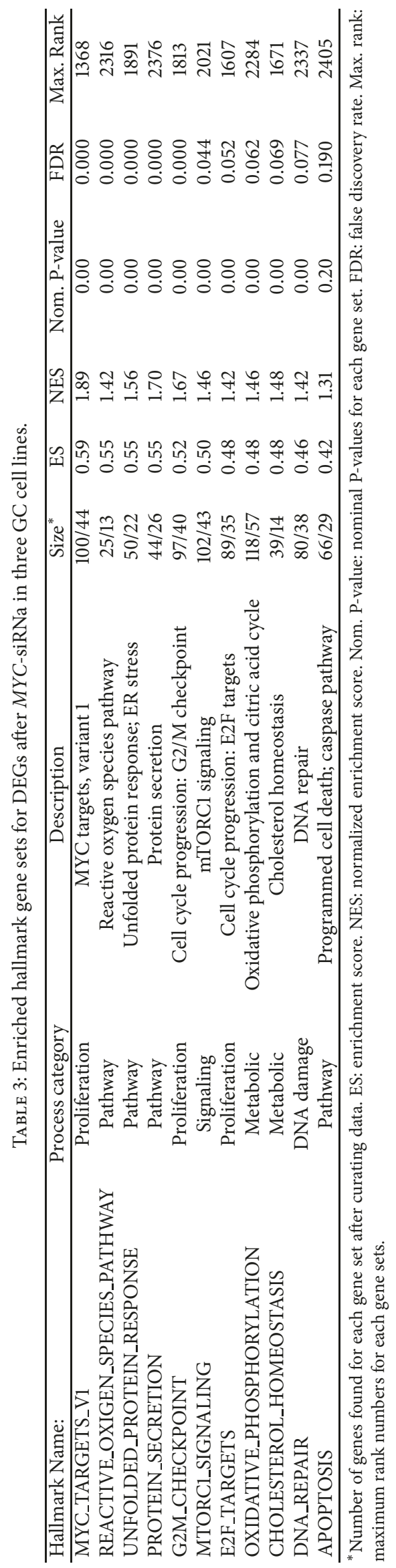




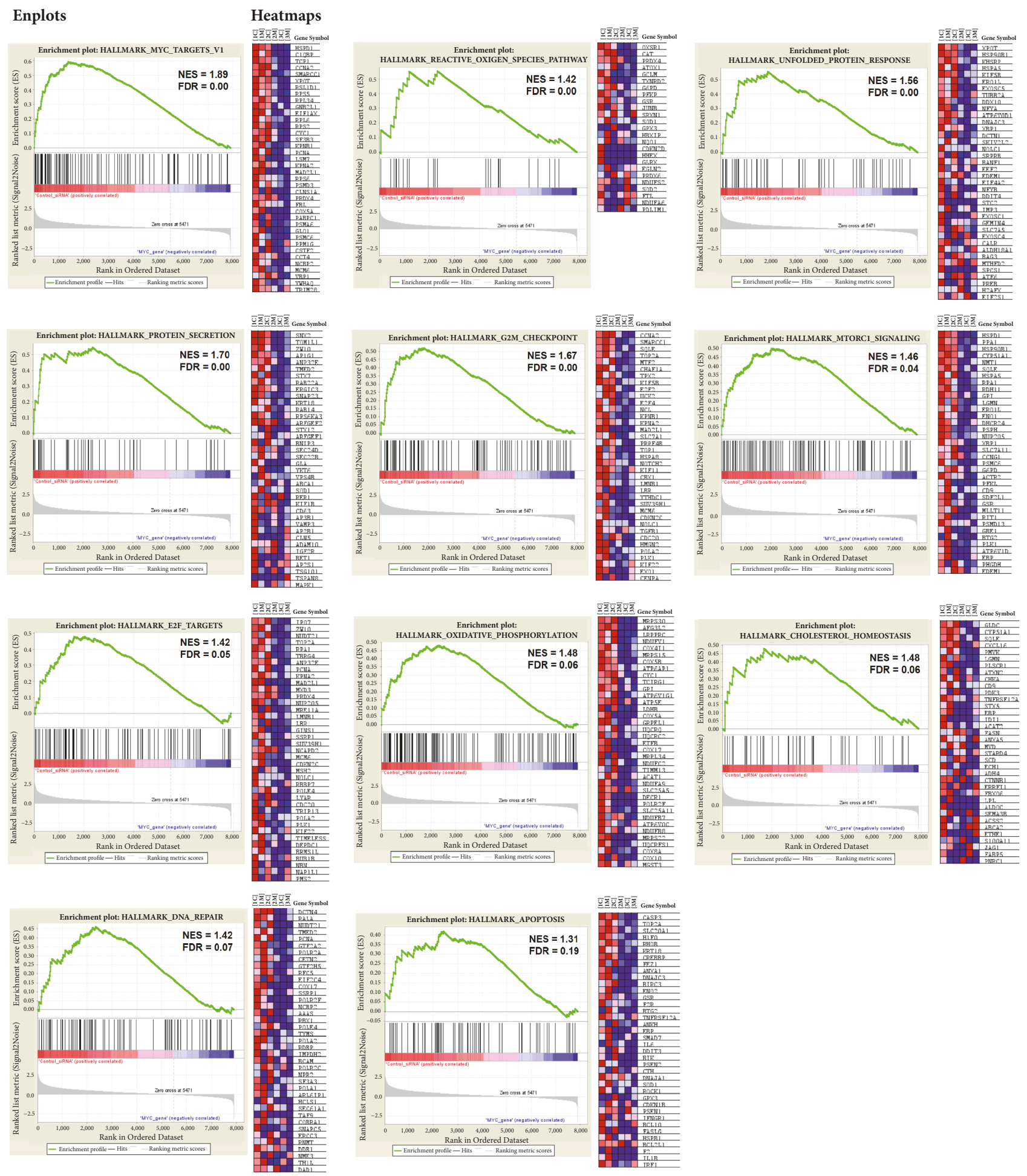

FIGURE 2: Panel with 11 gene sets enriched for three GC cell lines after $M Y C$-silencing. Each gene set was represented on the Enplot by its normalized enrichment score (NES) and False Discovery Rate (FDR), along with its heatmap showing the gene expression for each gene.

The enrichment maps obtained are shown in Figure 2, where we created a panel of 11 gene sets significantly enriched.

3.2. MYC-Silenced GC Cells Lines from Different Histological Subtypes Show Distinct MYC-Dependent Expression Profiles. We then evaluated the metabolic pathways more likely to be $M Y C$-regulated as shown by GSEA, listing those genes in the categories described as hallmarks of gene expression [40] and found that they are related to cellular proliferation, pathway, cellular signaling, metabolic processes, and response to DNA 
TABLE 4: The 14 common DEGs for three GC cell lines after MYC-siRNA and the gene set hallmarks they were enriched in.

\begin{tabular}{|c|c|c|c|c|}
\hline Hallmark Name: & Gene Symbol & Rank in Gene List & Rank Metric Score & Running (ES) \\
\hline PROTEIN_SECRETION & BNIP3 & 1438 & $6.0 \mathrm{E}-01$ & 0.522 \\
\hline UNFOLDED_PROTEIN_RESPONSE & SKIV $2 L 2$ & 1051 & $7.2 \mathrm{E}-01$ & 0.508 \\
\hline UNFOLDED_PROTEIN_RESPONSE & $S R P R B$ & 1442 & $6.0 \mathrm{E}-01$ & 0.506 \\
\hline REACTIVE_OXIGEN_SPECIES_PATHWAY & $J U N B$ & 1969 & $4.8 \mathrm{E}-01$ & 0.491 \\
\hline G2M_CHECKPOINT & $L B R$ & 817 & $8.2 \mathrm{E}-01$ & 0.451 \\
\hline OXIDATIVE_PHOSPHORYLATION & NDUFV2 & 1542 & $5.7 \mathrm{E}-01$ & 0.444 \\
\hline APOPTOSIS & $C D K N 1 B$ & 2405 & $4.0 \mathrm{E}-01$ & 0.420 \\
\hline PROTEIN_SECRETION & $R A B 22 A$ & 289 & $1.2 \mathrm{E}+15$ & 0.408 \\
\hline CHOLESTEROL_HOMEOSTASIS & ATXN2 & 945 & 7.6E- 01 & 0.382 \\
\hline CHOLESTEROL_HOMEOSTASIS & $L G M N$ & 445 & $1.1 \mathrm{E}+16$ & 0.367 \\
\hline OXIDATIVE_PHOSPHORYLATION & ACAT1 & 840 & $8.1 \mathrm{E}-01$ & 0.367 \\
\hline PROTEIN_SECRETION & TMED2 & 254 & $1.3 \mathrm{E}+16$ & 0.326 \\
\hline DNA_REPAIR & $A A A S$ & 1109 & 7.0E-01 & 0.313 \\
\hline G2M_CHECKPOINT & $N C L$ & 328 & $1.2 \mathrm{E}+13$ & 0.289 \\
\hline
\end{tabular}

ES: enrichment score.

damage (Table 3). We refined the raw data sets obtained from our GC cell lines, identifying the DEGs for each of the 11 gene sets for collection $\mathrm{H}$ that were enriched for the Control_siRNA phenotype. Figure 3 shows the ranks for enriched DEGs when compared to collection $\mathrm{H}$ with their average enrichment score (ES) for each analysis. Part of these results generated in this analysis is in Table 1 (see Supplementary Materials), which show the most enriched DEGs in the set gene MYC TARGETS V1.

The diffuse-type cell line (ACP02) presented 149 enriched DEGs by GSEA, 3 up- and 146 downregulated. Genes enriched with ranks above 15.000 fit the downregulated profile and were emphasized for gene sets such as MYC target V1, protein secretion, and reactive oxygen species pathway (Figure 3(a)). On the other hand, ranks above 30.000 were enriched for downregulated DEGs, presenting gene sets described as protein secretion, MYC target V1, unfolded protein response, and reactive oxygen species pathway (Figure 3(b)). Meanwhile, the intestinal-type cells (ACP03) showed 85 DEGs, 76 up- and 9 downregulated; genes with ranks above 20.000 were upregulated for pathways such as protein secretion, $M Y C$ target $\mathrm{V} 1$, and reactive oxygen species pathway (Figure 3(c)); downregulated genes with ranks above 15.000 presented enrichment emphasis for GM2-checkpoint, protein secretion, $M Y C$ target $\mathrm{V} 1$, reactive oxygen species pathway, and unfolded protein response (Figure 3(d)). For the metastatic samples (AGP01), 65 enriched DEGs were identified by GSEA, 25 up- and 40 downregulated, and the gene sets were enriched with ranks above 20.000 for both DEGs profiles. Upregulated sets (Figure 3(e)) were enriched for GM2-checkpoint, protein secretion, MYC target V1, and reactive oxygen species pathway, while the downregulated profile had emphasis for Oxidative_Phosphorylation, MYC target V1, protein secretion, and reactive oxygen species pathway (Figure 3(f)).

In the 11 gene sets from collection $\mathrm{H}$ that were used for DEGs enrichment, we identified the genes with higher ES. We used this strategy to identify similar genes that overlap as a consensus grouping as described elsewhere [40], so it would be easier to identify $M Y C$-regulated genes. We identified DEGs for the three GC cell lines that were enriched when compared to collection $\mathrm{H}$, with ranks above 2.000. The MYC target V1 gene set was identified as the most enriched for our studied cell lines, with higher ES scores for the genes SNRPD2 and TYMS. When we compared the enriched genes found for collection $\mathrm{H}$ for ACP02 and ACP03, we noticed that some genes presented enrichment ranks that were also common for the metastatic cells (AGP01) (Figures 4(a)-4(c)).

The same analysis was applied to identify enriched DEGs found for KEGG pathways, to deepen our understanding about the genes involved in the MYC-related carcinogenesis for the stomach. The enrichment maps for KEGG [42] are shown in Figures 4(d)-4(f), presenting 12 significantly enriched pathways found using GSEA, suggesting that GC has multiple altered pathways that lead normal gastric mucosa into the carcinogenic process. These pathways were defined by a normalized enrichment score (NES) $>1.47$. The average t-statistic for the genes was calculated for each KEGG pathway using permutation tests with 100 repetitions. The enrichment plot for three common pathways altered in all our cell lines is presented in Figure 4(g) (ribosomehsa03010), which presented the highest ES among the cells, Figure 4(h) (ubiquitin-mediated proteolysis-hsa04120), with the highest ranks in our gene list, and Figure 4(i) (epithelial cell signaling in Helicobacter pylori infection-hsa05120). For more details on the DEGs enriched with KEGG, see Table 2 in Supplementary Materials.

We identified enriched DEGs common to all three GC cell lines used in this study and represent them as Venn diagrams constructed by the InteractiVenn platform [43]. We found 14 DEGs that are shared enriched in both AGP01, ACP02, and ACP03 cell lines (Figure 5(a)), which are likely to be $M Y C$ targets according to our analysis. When we search for gene function in different databases (Figure 5(b)), most of them $(14.78 \%)$ are involved in protein secretion, followed by unfolded protein response $(14.39 \%)$ and reactive oxygen species pathway (13.91\%). Table 4 shows the 14 individual 


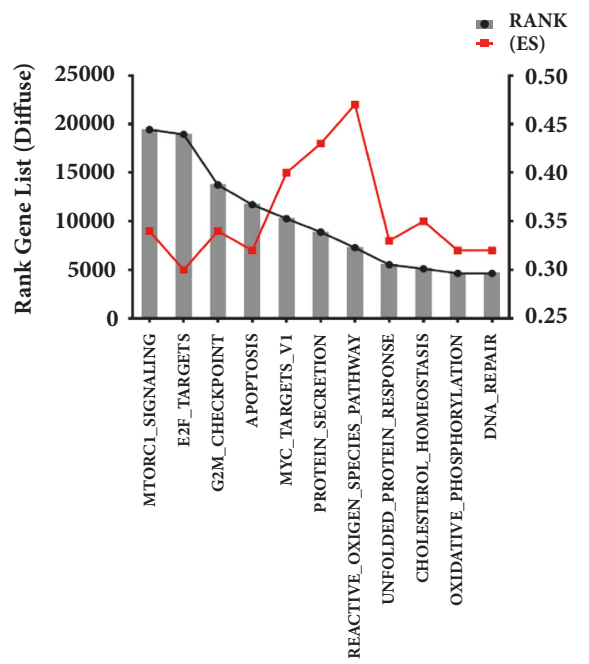

(a)

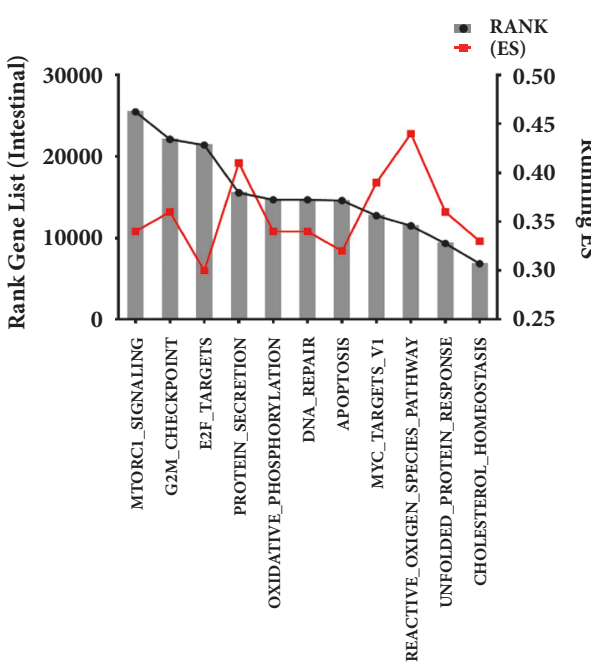

(c)

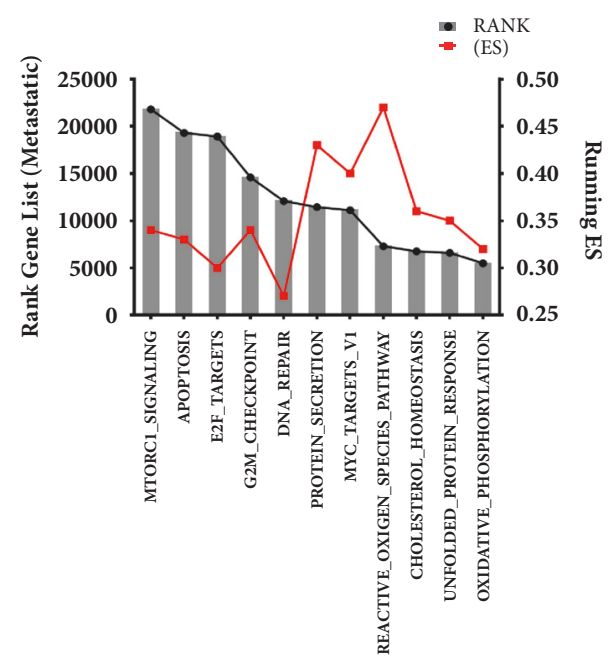

(e)

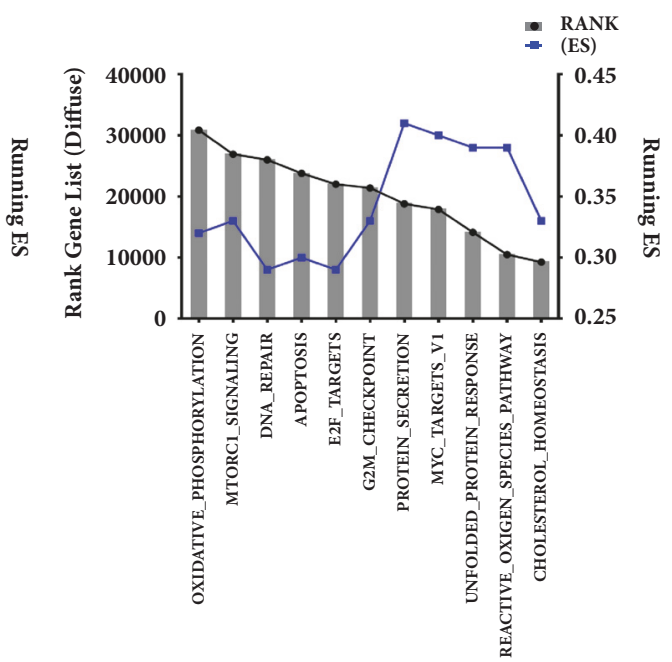

(b)

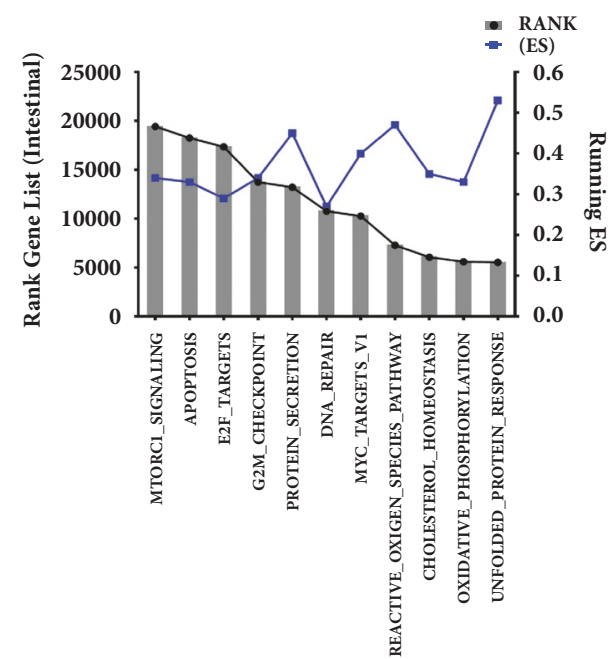

(d)

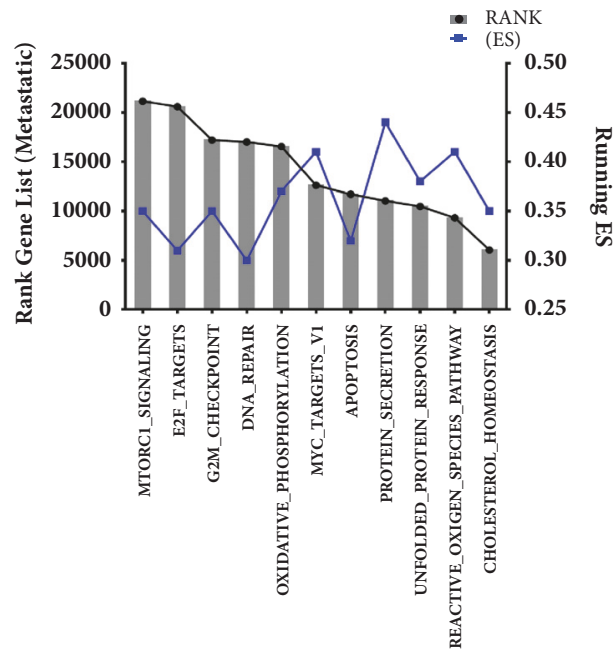

(f)

FIGURE 3: Ranks for enriched DEGs when compared to collection H with their average enrichment score (ES) for each analysis. (a) ACP02 upregulated DEGs, ranks (4.640 to 19.433), and ES (0.30 to 0.47). (b) ACP02 downregulated DEGs, ranks (9.245 to 30.875 ), and ES (0.29 to 0.41). (c) ACP03 upregulated DEGs, ranks (6.853 to 25.475), and ES (0.30 to 0.47). (d) ACP03 downregulated DEGs, ranks (5.530 to 19.433), and ES (0.27 to 0.53). (e) AGP01 upregulated DEGs, ranks (5.480 to 21.804), and ES (0.27 a 0.47). (f) AGP01 downregulated DEGs, ranks (6.060 to 21.134$)$, and ES (0.30 a 0.44$)$. 


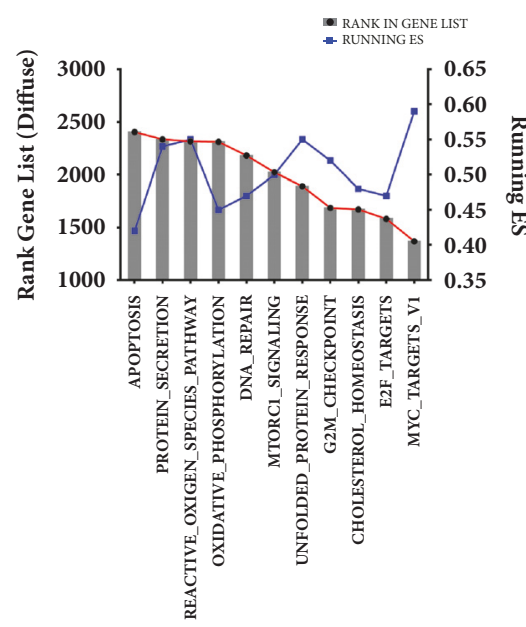

(a)

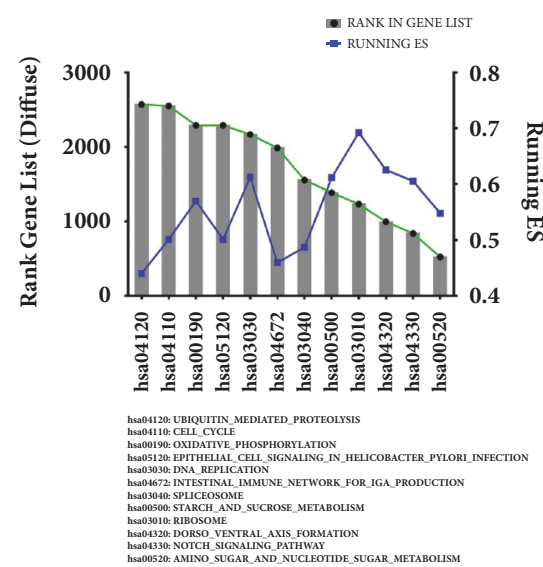

(d)

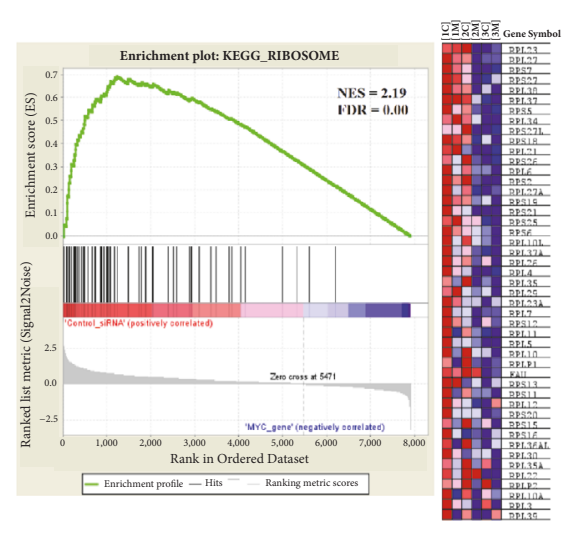

(g)

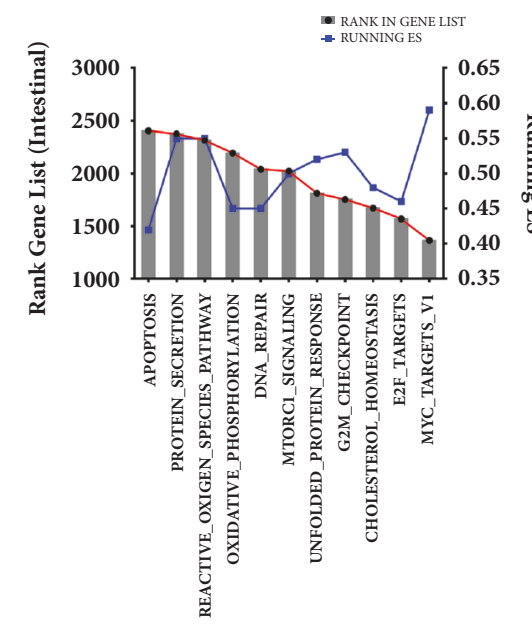

(b)

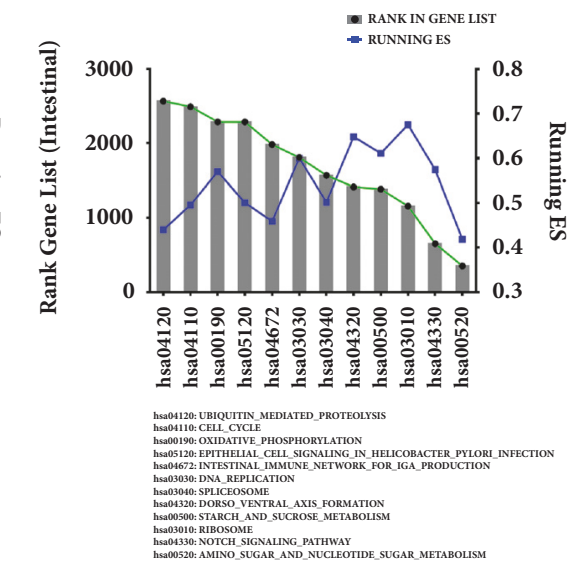

(e)

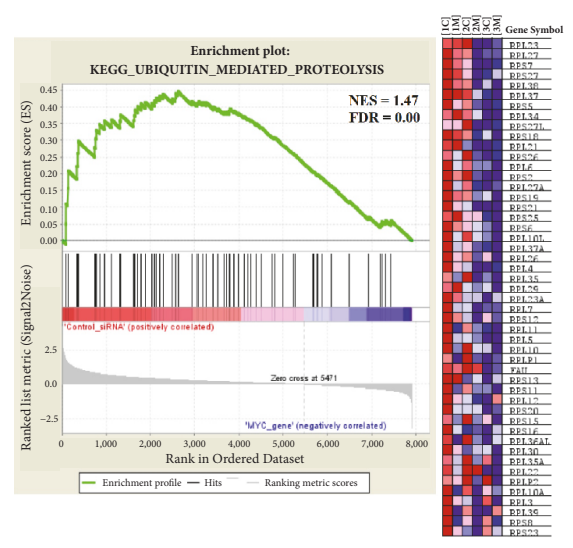

(h)

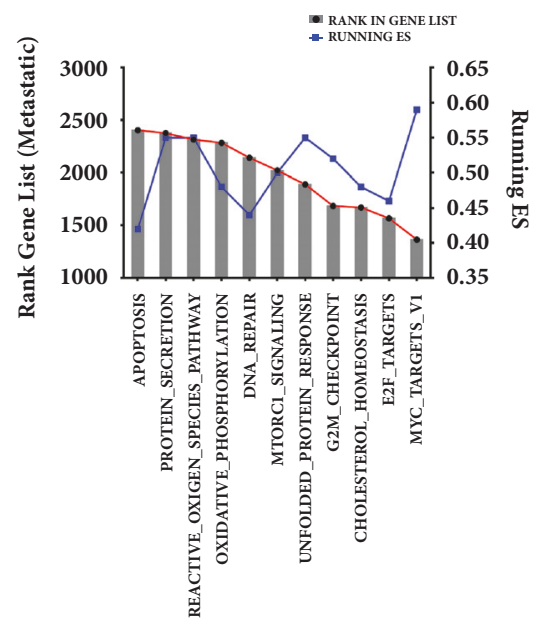

(c)

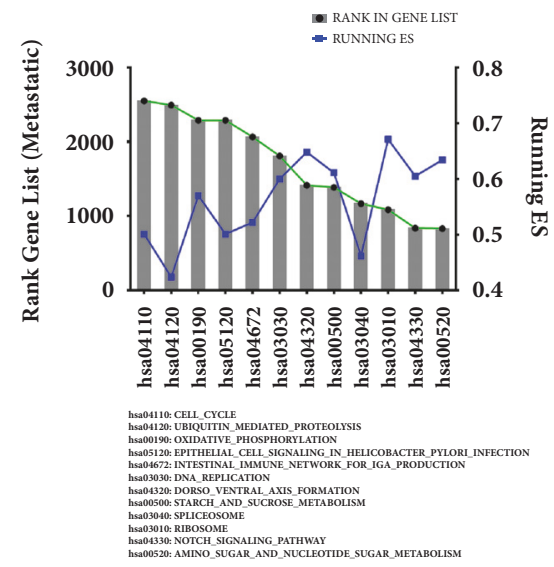

(f)

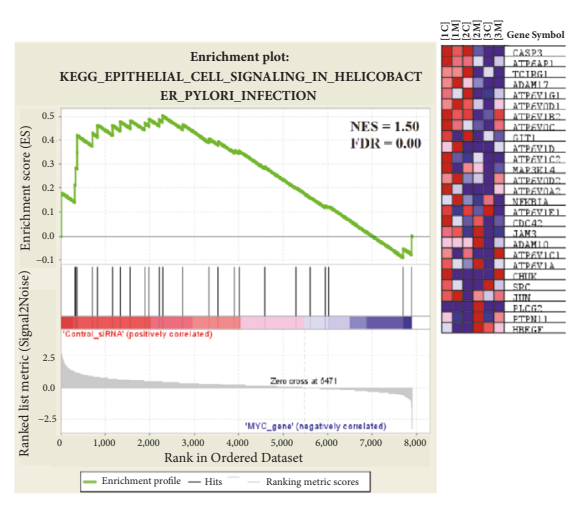

(i)

FIGURE 4: Identification of individual genes in the enriched DEG datasets with the higher ES. (a) DEGs enrichment for ACP02. (b) DEGs enrichment for ACP03. (c) DEGs enrichment for AGP01. The most enriched hallmark for the three cell lines as MYC_Target_V1 with an ES = 0.59. (d) KEGG pathway identification for ACP02. (e) KEGG pathway identification for ACP03. (f) KEGG pathway identification for AGP01. Enplot and heatmap for the pathways with the highest ES scores among the three GC cell lines can be seen in (g) for ACP02, in (h) for ACP03, and in (i) for AGP01. 


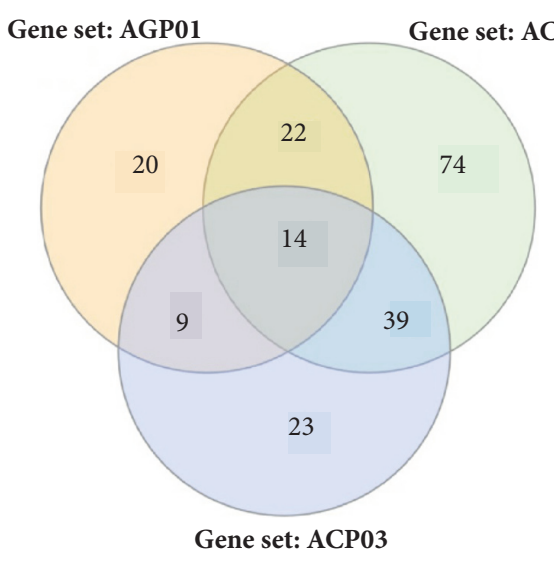

(a)

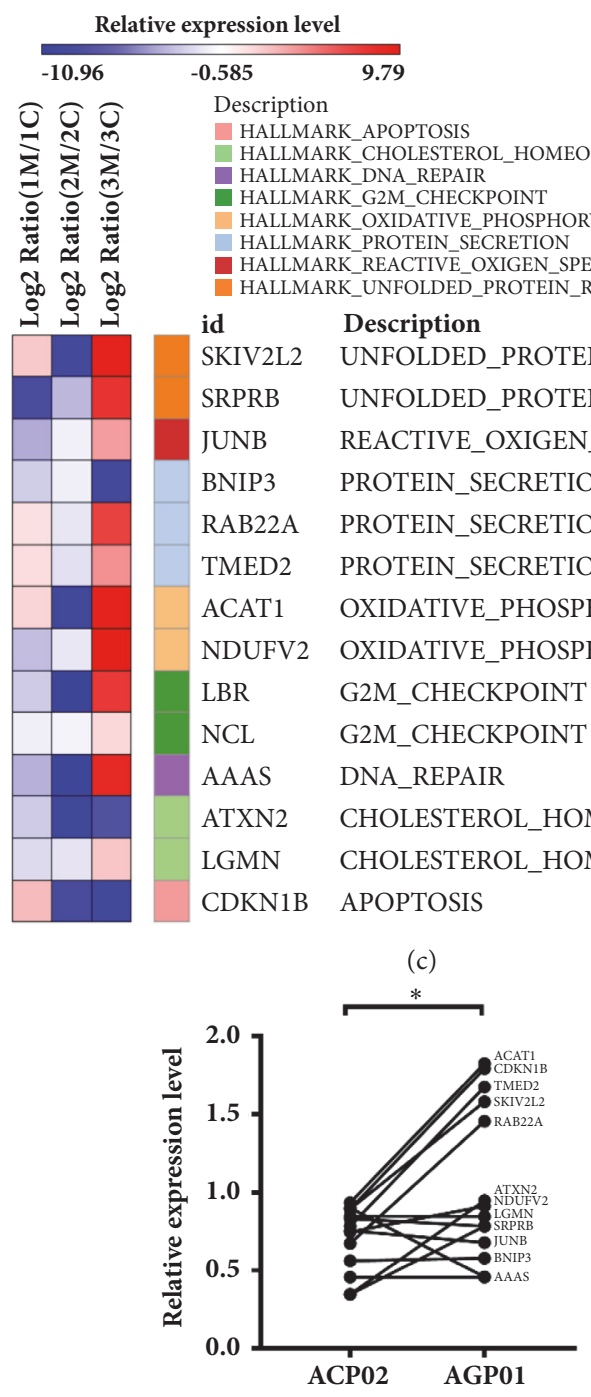

(e)

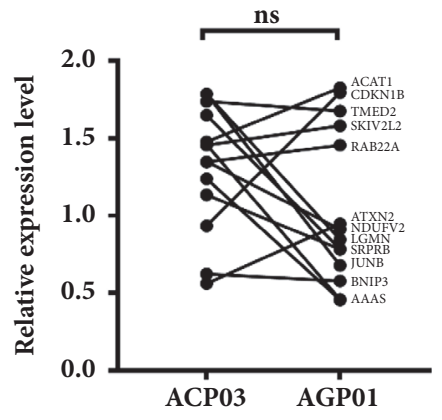

(f)

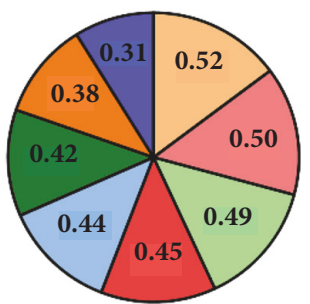

14.78\% PROTEIN SECRETION

$\square 14.39 \%$ UNFOLDED PROTEIN RESPONSE

13.91\% REACTIVE OXIGEN SPECIES PATHWAY

$12.77 \%$ G2M CHECKPOINT

$12.57 \%$ OXIDATIVE PHOSPHORYLATION

$11.89 \%$ APOPTOSIS

$10.82 \%$ CHOLESTEROL HOMEOSTASIS

$8.86 \%$ DNA REPAIR

(b)

Relative expression level (Normal gastric mucosa cell line MN01)
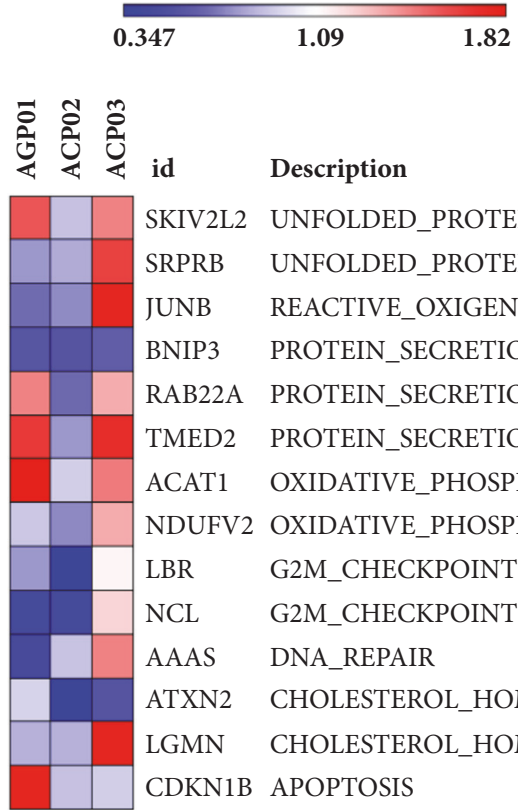

id Description

SKIV2L2 UNFOLDED_PROTEIN_RESPONSE

SRPRB UNFOLDED_PROTEIN_RESPONSE

JUNB REACTIVE_OXIGEN_SPECIES_PATHWAY

BNIP3 PROTEIN_SECRETION

RAB22A PROTEIN_SECRETION

TMED2 PROTEIN_SECRETION

ACAT1 OXIDATIVE_PHOSPHORYLATION

NDUFV2 OXIDATIVE_PHOSPHORYLATION

LBR G2M_CHECKPOINT

NCL G2M_CHECKPOINT

AAAS DNA_REPAIR

ATXN2 CHOLESTEROL_HOMEOSTASIS

LGMN CHOLESTEROL_HOMEOSTASIS

CDKN1B APOPTOSIS

(d)

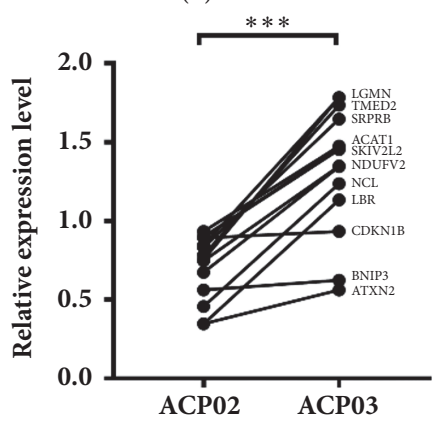

(g)

FIGURE 5: Common DEGs enriched in three GC cell lines after $M Y C$-silencing. (a) Using Venn diagram, we were able to identify 14 common DEGs among AGP01, ACP02, and ACP03, but each one of them also has a unique set of DEGs. (b) Identification of the 14 common DEGs in 8 enriched hallmarks of collection $\mathrm{H}$, represented by the \% of its correspondent ES, ES scores are shown inside the pie chart for each hallmark. (c). Heatmap for the expression of the 14 common DEGs shared for three GC cell lines after MYC-silencing. Blue represents downregulation while red means upregulation. Notice that, even for the 14 common genes, each cell line has a different expression pattern. Relative read expression was normalized using $\log _{2}$ Fold-change between $M Y C$-silenced/control siRNA. (d) Heatmap for the relative gene expression obtained by RT-qPCR for the 14 identified DEGS for the AGP01, ACP02, and ACP03 cell lines. (e) The overall expression for the 14 DEGs is downregulated in the ACP02 cell line when compared to AGP01 cells. (f) The gene expression levels were increased in ACP03 cell line, but no statistical difference was found. (g) Relative gene expression was increased for ACP03 when compared to ACP02. Wilcoxon matched-pairs signed rank test was used to compare the relative gene expression levels $\left({ }^{*} \mathrm{P}<0.05\right.$, ns: not significant, and $\left.{ }^{* * *} \mathrm{P}<0.0001\right)$. 
DEGs as well as the gene expression hallmarks they are involved, ranks and ES. We show a heatmap (Figure 5(c)) of the 14 shared DEGs in clusters grouped using the GENE E tool (https://software.broadinstitute.org/GENE-E/), where downregulation is expressed in blue and upregulation in red. Most genes for ACP02 (diffuse-type GC) presented downregulation, while for ACP03 (intestinal-type GC) the same genes presented themselves as upregulated. The metastatic cell line AGP01, even though its original tumor was intestinaltype, presented mixed expression patterns with predominant downregulation.

\subsection{The 14 MYC-Regulated DEGs Show Distinct Expression} Profiles for Each GC Histological Subtype. Our in silico analysis identified a core set of 14 DEGs (Figure 5(a)) who are $M Y C$-regulated, but whose expression profile is distinct for each GC cell line since they represent different histological subtypes. We validated the gene expression profiles presented by the AGP01, ACP02, and ACP03 after MYC-silencing by RT-qPCR in the cDNA obtained originally for each cell line and compared them with their expression in the MN01 cell line. Our results point out that the 14 identified DEGs are under MYC transcriptional regulation (Figure 5(d)). The ACP02 cell line presented mostly downregulation for the expression of the 14 DEGs (Figure 5(c)); on the other hand, ACP03, the same 14 genes, were upregulated; AGP01 results were mixed, with some genes showing downregulation and others showing downregulation. Our gene expression results (Figure 5(d)) confirm our in silico analysis (Figure 5(c)).

Each cell line used in this study represents a GC histological subtype: AGP01 was obtained from the ascitic fluid of intestinal-type GC, representing a metastatic disease, while ACP02 was developed from a diffuse-type stomach cancer patient and ACP03 origin was an intestinal-type gastric tumor.

We compared the mRNA expression measured by RTqPCR for our identified DEGs to assess whether the gene expression of those 14 genes was enough to statistically distinguish each cell line. When comparing ACP02 versus AGP01 (Figure 5(e)), we noticed a significant gene expression downregulation for ACP02; confronting ACP03 versus AGP01 indicated an increase in mRNA relative quantification (Figure 5(f)) for ACP03; however, those results were not significant; the comparison between ACP02 versus ACP03 confirmed that the 14 MYC-regulated DEGs are significantly more expressed in the ACP03, the intestinal-type cell line, than in ACP02 (Figure 5(g)).

Taken together, our results indicate that, even though MYC-related carcinogenesis alters the same 14 genes in GC cell lines representing the most common histological subtypes, how MYC causes GC carcinogenesis is different for each disease presentation and it is possible to distinct them by using expression signatures.

\section{Discussion}

A key goal of cancer studies is to systematically characterize the cellular and molecular mechanisms involved in the disease and its distinct stages, to identify both potential biomarkers and new probable drug targets [44]. The molecular profile of gastric cancer is heterogeneous, partly due to different classification systems [45] and, in order to clarify the true molecular origins of GC, both the Cancer Genome Atlas [22] and the Asian Cancer Research Group [46] published the molecular subtypes of gastric cancer, with remarkable overlap between the two models. Therefore, several genes have been implied as biomarkers for GC subtypes, such as RHOA, EGFR, PDL, CDH1, TP53, and JAK2. However, those studies use samples from populations in which the disease incidence is highest, and few studies have examined populations in which the incidence of this disease is lower, such as Brazil [47]. There is evidence that GC incidence varies between countries greatly because the genetic heterogeneity exhibited by human populations [48], and it has already been showed that there is a unique gene expression signature for Brazilian cases of intestinal-type GC [47]. Our study helps to highlight the molecular profiles of Brazilian GC cell lines, which can help greatly our understanding about the molecular basis of GC in South America.

Most NGS studies investigate GC by comparing tumor versus nontumoral tissue, analyzing global gene expression patterns, copy number variation, and other molecular characteristics, and most of the high-throughput studies carried so far concerning gastric cancer genetics overlook MYC's importance in this process [2, 3, 21-24]. Our results are relevant because MYC overexpression is a key finding in Brazilian GC samples [8]. Therefore, we reduced the expression of this gene using siRNA to identify the MYC-related signature in GC cell lines, comparing nonsilenced with silenced samples. We identified a total of 5.471 DEGs, and 11 significant gene sets, including classic MYC targets represented by MYC target V1.

We hereby present the computational analysis of gene sets identified after MYC-silencing in Brazilian GC cell lines $[13,27]$, who carry genetic alterations commonly found in Brazilian GC patients [7, 13, 16]. This oncogene promotes cell growth acting as a transcription factor regulating cell cycle, metabolism, and cell survival [49]. We found DEGs upregulation only for the intestinal-type cell line (ACP03), while the diffuse-type (ACP02) and the metastatic GC cells (AGP01) presented overall gene expression downregulation; when looking at individual genes between ACP02 and AGP01, it is still possible to distinguish between them by $M Y C$-related gene expression. It is important to notice that $M Y C$ has a dual-role in the carcinogenic process, selectively activating and inactivating different gene sets [50-52]. Taken together, we present evidences supporting the fact that MYC deregulation has an important role in gastric carcinogenesis [14] and that $M Y C$-related signatures in gastric cancer are different for each histological subtype of this disease, which is clinically relevant $[47,53]$.

One of the main forms of MYC protein regulation in normal cells is through its targeted degradation by the ubiquitin-proteasome system [54], which was one of the KEGG enriched pathways found in our analysis (Figure 4). This means that, in a MYC-overexpression condition, like GC [7], not only this gene and its protein are more produced, but they are also less destroyed because it diminishes the expression of E3 ubiquitin-ligases, such as Fbw7 and HectH9, 
contributing to prolonged MYC protein half-life and amplification of its effects [55]. Ubiquitin-ligases, including the MYC-regulated Fbw7, have recently evolved as promising therapeutic targets for the development of novel anticancer drugs [56].

Other pathways involved in MYC-related gastric carcinogenesis found by our study are known targets, such as ribosome and cell cycle control genes, which are hardly druggable. When taken together, the 12 different pathways we found under MYC control for gastric carcinogenesis represent many biological functions, meaning that $M Y C$ overexpression in GC disturbs almost all the regular cellular processes in favor of tumor development [52]. Another interesting gene set includes glucose metabolism, which is an area of growing interest in cancer research [57], and it has been shown that MYC directs the activation of aerobic glycolysis, a hallmark of cancer metabolism known as Warburg effect, and pretty much all genes involved in glycolysis and most of the ones responsible for glutaminolysis [57, 58].

We were also able to pinpoint 14 enriched DEGs in all the three GC cell lines used in this study that might represent the common set of MYC-regulated genes in gastric carcinogenesis (Table 4). This is important because it shows that, even though we have represented distinct GC histological subtypes and disease stages, there is still a core set of genes regulated by MYC involved in the carcinogenic process. We also did not find other reports in the literature concerning those 14 genes and high-throughput analysis of gastric cancer $[1,22,26,44-46,53,59-64]$. Even when we consider the molecular signatures presented by Brazilian intestinal-type GC [47], we could not find any concordance for the 14 genes found by our study, but it is important to consider that Binato et al. [47] did not take into account MYC overexpression in their samples. Therefore, the unique DEGs found in this paper represent new and important findings concerning the process of gastric carcinogenesis regulated by MYC in the Brazilian population.

Even though additional studies are needed to validate our results, we present strong evidence that MYC-regulated genes in GC have different expression patterns when we consider histological and disease stage differences; however, they still share pathways and core genes involved in the carcinogenic process.

\section{Data Availability}

The gene expression data used to support the findings of this study have been deposited in the Gene Expression Omnibus (GEO) repository under the access number GSE81265 (https:// www.ncbi.nlm.nih.gov/geo/query/acc.cgi?acc=GSE81265). A detailed description of each gene set can be found within the paper at Table 1 .

\section{Conflicts of Interest}

The authors declare that there are no conflictd of interest regarding the publication of this paper.

\section{Authors' Contributions}

Jersey Heitor da S. Maués, Helem Ferreira Ribeiro, Caroline de Fátima Aquino Moreira-Nunes, and Giovanny R. Pinto conceived and designed the study. Helem Ferreira Ribeiro, Luana de Oliveira Lopes, Giovanny R. Pinto, Letícia M. Lamarão, and Carla Mariana F. Pessoa conducted the study and participated in laboratory analysis. Raimundo Miranda de Carvalho, Jersey Heitor da S. Maués, Helem Ferreira Ribeiro, Juan A. Rey, and Paulo P. Assumpção conducted the RT-qPCR analysis. Jersey Heitor da S. Maués, Caroline de Fátima Aquino Moreira-Nunes, and Rommel M. Rodríguez Burbano conducted bioinformatic analysis and analyzed the data. Jersey Heitor da S. Maués, Helem Ferreira Ribeiro, and Rommel M. Rodríguez Burbano wrote the paper. All authors read and approved the final manuscript.

\section{Acknowledgments}

This work was supported by CNPq, Process no. 402283/2013 and Process no. 471072/2012-5; by FAPESPA, Process no. ICAAF 123/2014; by CAPES, Process no. 17704/12-0.

\section{Supplementary Materials}

Table 1: enriched hallmark gene sets for DEGs. Table 2: enriched KEGG gene sets for DEGs. Table 3: the 14 common DEGs for three GC cell lines after MYC siRNA and the gene set hallmarks they were enriched and identified in cancerassociated studies. (Supplementary Materials)

\section{References}

[1] B. W. Katona and A. K. Rustgi, "Gastric cancer genomics: advances and future directions," Cellular and Molecular Gastroenterology and Hepatology, vol. 3, no. 2, pp. 211-217, 2017.

[2] M. H. McLean and E. M. El-Omar, "Genetics of gastric cancer," Nature Reviews Gastroenterology \& Hepatology, vol. 11, no. 11, pp. 664-674, 2014.

[3] K. Wang, S. T. Yuen, J. Xu et al., "Whole-genome sequencing and comprehensive molecular profiling identify new driver mutations in gastric cancer," Nature Genetics, vol. 46, no. 6, pp. 573-582, 2014.

[4] A. D. Panani, "Cytogenetic and molecular aspects of gastric cancer: Clinical implications," Cancer Letters, vol. 266, no. 2, pp. 99-115, 2008.

[5] X. Liu, H. Cai, H. Huang et al., "The prognostic significance of apoptosis-related biological markers in chinese gastric cancer patients," PLoS ONE, vol. 6, no. 12, 2011.

[6] J.-S. Choi, J. Seo, E. J. Jung, E. J. Kim, G. K. Lee, and W. H. Kim, "c-MYC amplification in mucinous gastric carcinoma: A possible genetic alteration leading to deeply invasive tumors," Anticancer Reseach, vol. 32, no. 11, pp. 5031-5038, 2012.

[7] D. Q. Calcagno, V. M. Freitas, M. F. Leal et al., "MYC, FBXW7 and TP53 copy number variation and expression in Gastric Cancer," BMC Gastroenterology, vol. 13, no. 1, 2013.

[8] M. F. Leal, H. F. Ribeiro, J. A. Rey et al., "YWHAE silencing induces cell proliferation, invasion and migration through the up-regulation of CDC25B and MYC in gastric cancer cells: New insights about YWHAE role in the tumor development and 
metastasis process," Oncotarget, vol. 7, no. 51, pp. 85393-85410, 2016.

[9] A. A. Mello, M. F. Leal, J. A. Rey et al., "Deregulated expression of SRC, LYN and CKB kinases by DNA methylation and its potential role in gastric cancer invasiveness and metastasis," PLoS ONE, vol. 10, no. 10, 2015.

[10] W. P. Tansey, "Mammalian MYC Proteins and Cancer," New Journal of Science, vol. 2014, Article ID 757534, 27 pages, 2014.

[11] L. C. Costa Raiol, E. C. Figueira Silva, D. Mendes da Fonseca et al., "Interrelationship between MYC gene numerical aberrations and protein expression in individuals from northern Brazil with early gastric adenocarcinoma," Cancer Genetics and Cytogenetics, vol. 181, no. 1, pp. 31-35, 2008.

[12] D. Q. Calcagno, M. F. Leal, S. Demachki et al., "MYC in gastric carcinoma and intestinal metaplasia of young adults," Cancer Genetics and Cytogenetics, vol. 202, no. 1, pp. 63-66, 2010.

[13] M. F. Leal, D. Q. Calcagno et al., "MYC, TP53, and chromosome 17 copy-number alterations in multiple gastric cancer cell lines and in their parental primary tumors," Journal of Biomedicine and Biotechnology, vol. 2011, Article ID 631268, 8 pages, 2011.

[14] C. R. T. De Souza et al., "MYC deregulation in gastric cancer and its clinicopathological implications," PLoS One, vol. 8, no. 5, 2013.

[15] J. D. F. F. Borges Da Costa et al., "Experimental gastric carcinogenesis in cebus apella nonhuman primates," PLoS One, vol. 6, no. 7, p. 13, 2011.

[16] F. Wisnieski, D. Q. Calcagno, M. F. Leal et al., "Reference genes for quantitative RT-PCR data in gastric tissues and cell lines," World Journal of Gastroenterology, vol. 19, no. 41, pp. 7121-7128, 2013.

[17] J. R. Whitfield and L. Soucek, "Tumor microenvironment: Becoming sick of Myc," Cellular and Molecular Life Sciences, vol. 69, no. 6, pp. 931-934, 2012.

[18] S. A. Hills and J. F. X. Diffley, "DNA replication and oncogeneinduced replicative stress," Current Biology, vol. 24, no. 10, pp. R435-R444, 2014.

[19] L. Zhang, Y. Hou, H. Ashktorab et al., "The impact of C-MYC gene expression on gastric cancer cell," Molecular and Cellular Biochemistry, vol. 344, no. 1-2, pp. 125-135, 2010.

[20] S. Walz and M. Eilers, "Unlocking the mysterious mechanisms of Myc," Nature Medicine, vol. 19, no. 1, pp. 26-27, 2013.

[21] P. Karimi, F. Islami, S. Anandasabapathy, N. D. Freedman, and F. Kamangar, "Gastric cancer: descriptive epidemiology, risk factors, screening, and prevention," Cancer Epidemiology Biomarkers \& Prevention, vol. 23, no. 5, pp. 700-713, 2014.

[22] A. J. Bass et al., "Comprehensive molecular characterization of gastric adenocarcinoma," Nature, vol. 513, no. 7517, pp. 202-209, 2014.

[23] C. Figueiredo, M. C. Camargo, M. Leite, E. M. Fuentes-Pananá, C. S. Rabkin, and J. C. Machado, Pathogenesis of Gastric Cancer: Genetics and Molecular Classification BT - Molecular Pathogenesis and Signal Transduction by Helicobacter pylori, N. Tegtmeyer and S. Backert, Eds., vol. 400 of Current Topics in Microbiology and Immunology, Springer International Publishing, 2017.

[24] J. Cools-Lartigue, L. Baker, and L. E. Ferri, Molecular Mechanisms in Gastric Carcinogenesis BT - Gastric Cancer: Principles and Practice, V. E. Strong, Ed., Springer International Publishing, 2015.

[25] M. Kato, "Bioinformatics in cancer clinical sequencing-an emerging field of cancer personalized medicine," Gan To Kagaku Ryoho, vol. 43, no. 4, pp. 391-397, 2016.
[26] C. Sun, Q. Yuan, D. Wu, X. Meng, and B. Wang, "Identification of core genes and outcome in gastric cancer using bioinformatics analysis," Oncotarget, vol. 8, no. 41, pp. 70271-70280, 2017.

[27] M. F. Leal, J. L. Martins do Nascimento, C. E. A. da Silva et al., "Establishment and conventional cytogenetic characterization of three gastric cancer cell lines," Cancer Genetics and Cytogenetics, vol. 195, no. 1, pp. 85-91, 2009.

[28] A. S. Khayat, A. C. Guimarães, D. Q. Calcagno et al., "Interrelationship between TP53 gene deletion, protein expression and chromosome 17 aneusomy in gastric adenocarcinoma," BMC Gastroenterology, vol. 9, no. 1, 2009.

[29] H. F. Ribeiro, D. F. A. Alcântara, L. A. Matos et al., "Cytogenetic characterization and evaluation of c-MYC gene amplification in PG100, a new Brazilian gastric cancer cell line," Brazilian Journal of Medical and Biological Research, vol. 43, no. 8, pp. 717-721, 2010.

[30] H. Li and R. Durbin, "Fast and accurate long-read alignment with Burrows-Wheeler transform," Bioinformatics, vol. 26, no. 5, pp. 589-595, 2010.

[31] N. Leng, J. A. Dawson, J. A. Thomson et al., "EBSeq: an empirical Bayes hierarchical model for inference in RNA-seq experiments," Bioinformatics, vol. 29, no. 8, pp. 1035-1043, 2013.

[32] H. Li, "Exploring single-sample snp and indel calling with whole-genome de novo assembly," Bioinformatics, vol. 28, no. 14, Article ID bts280, pp. 1838-1844, 2012.

[33] R. Patro, S. M. Mount, and C. Kingsford, "Sailfish enables alignment-free isoform quantification from RNA-seq reads using lightweight algorithms," Nature Biotechnology, vol. 32, no. 5, pp. 462-464, 2014.

[34] C. Trapnell, B. A. Williams, G. Pertea et al., "Transcript assembly and quantification by RNA-Seq reveals unannotated transcripts and isoform switching during cell differentiation," Nature Biotechnology, vol. 28, no. 5, pp. 511-515, 2010.

[35] M. Garber, M. G. Grabherr, M. Guttman, and C. Trapnell, "Computational methods for transcriptome annotation and quantification using RNA-seq," Nature Methods, vol. 8, no. 6, pp. 469-477, 2011.

[36] S. Audic and J.-M. Claverie, "The significance of digital gene expression profiles," Genome Research, vol. 7, no. 10, pp. 986995, 1997.

[37] H. Abdi, “The Bonferonni and Šidák Corrections for Multiple Comparisons,” pp. 1-9, 2007.

[38] Y. Benjamini and D. Yekutieli, "The control of the false discovery rate in multiple testing under dependency," The Annals of Statistics, vol. 29, no. 4, pp. 1165-1188, 2001.

[39] A. Subramanian, P. Tamayo, V. K. Mootha, S. Mukherjee, and B. L. Ebert, "Gene set enrichment analysis: a knowledge-based approach for interpreting genome-wide," 2005.

[40] A. Liberzon, C. Birger, H. Thorvaldsdóttir, M. Ghandi, J. P. Mesirov, and P. Tamayo, "The molecular signatures database hallmark gene set collection," Cell Systems, vol. 1, no. 6, pp. 417425, 2015.

[41] A. Liberzon, A. Subramanian, R. Pinchback, H. Thorvaldsdóttir, P. Tamayo, and J. P. Mesirov, "Molecular signatures database (MSigDB) 3.0," Bioinformatics, vol. 27, no. 12, pp. 1739-1740, 2011.

[42] M. Kanehisa, S. Goto, Y. Sato, M. Furumichi, and M. Tanabe, "KEGG for integration and interpretation of large-scale molecular data sets," Nucleic Acids Research, vol. 40, no. 1, pp. D109D114, 2012.

[43] H. Heberle, V. G. Meirelles, F. R. da Silva, G. P. Telles, and R. Minghim, "InteractiVenn: a web-based tool for the analysis of 
sets through Venn diagrams," BMC Bioinformatics, vol. 16, no. 1, article 169, 2015.

[44] J. Y. Cho, "Molecular diagnosis for personalized target therapy in gastric cancer," Gastric Cancer, vol. 13, no. 3, pp. 129-135, 2013.

[45] M. Rugge, M. Fassan, and D. Y. Graham, "Epidemiology of gastric cancer," Gastric Cancer: Principles and Practice, pp. 2334, 2015.

[46] R. Cristescu, J. Lee, M. Nebozhyn et al., "Molecular analysis of gastric cancer identifies subtypes associated with distinct clinical outcomes," Nature Medicine, vol. 21, no. 5, pp. 449-456, 2015.

[47] R. Binato, E. C. Santos, M. Boroni, S. Demachki, P. Assumpção, and E. Abdelhay, "A common molecular signature of intestinaltype gastric carcinoma indicates processes related to gastric carcinogenesis," Oncotarget, vol. 9, no. 7, pp. 7359-7371, 2018.

[48] T. E. Buffart, M. Louw, N. C. van Grieken et al., "Gastric cancers of Western European and African patients show different patterns of genomic instability," BMC Medical Genomics, vol. 4, no. 1, 2011.

[49] V. Posternak and M. D. Cole, "Strategically targeting MYC in cancer," F1000Research, vol. 5, 2016.

[50] S. Walz, F. Lorenzin, J. Morton et al., "Activation and repression by oncogenic MYC shape tumour-specific gene expression profiles," Nature, vol. 511, no. 7510, pp. 483-487, 2014.

[51] T. R. Kress, A. Sabò, and B. Amati, "MYC: Connecting selective transcriptional control to global RNA production," Nature Reviews Cancer, vol. 15, no. 10, pp. 593-607, 2015.

[52] T. R. Kress, P. Pellanda, L. Pellegrinet et al., "Identification of MYC-dependent transcriptional programs in oncogeneaddicted liver tumors," Cancer Research, vol. 76, no. 12, pp. 3463-3472, 2016.

[53] S. Zang, R. Guo, R. Xing et al., "Identification of differentiallyexpressed genes in intestinal gastric cancer by microarray analysis," Genomics, Proteomics \& Bioinformatics, vol. 12, no. 6, pp. 276-283, 2014.

[54] A. S. Farrell and R. C. Sears, "MYC degradation," Cold Spring Harbor Perspectives in Medicine, vol. 4, no. 3, Article ID a014365, 2014.

[55] B. King, T. Trimarchi, L. Reavie et al., "The ubiquitin ligase FBXW7 modulates leukemia-initiating cell activity by regulating MYC stability," Cell, vol. 153, no. 7, pp. 1552-1566, 2013.

[56] J. Liu, S. Shaik, X. Dai et al., "Targeting the ubiquitin pathway for cancer treatment," Biochimica et Biophysica Acta (BBA) Reviews on Cancer, vol. 1855, no. 1, pp. 50-60, 2015.

[57] C. V. Dang, "MYC, metabolism, cell growth, and tumorigenesis," Cold Spring Harbor Perspectives in Medicine, vol. 3, no. 8, 2013.

[58] Z. E. Stine, Z. E. Walton, B. J. Altman, A. L. Hsieh, and C. V. Dang, "MYC, metabolism, and cancer," Cancer Discovery, vol. 5, no. 10, pp. 1024-1039, 2015.

[59] S. S. Wong, K. Kim, J. C. Ting et al., "Genomic landscape and genetic heterogeneity in gastric adenocarcinoma revealed by whole-genome sequencing," Nature Communications, vol. 5, no. 1, 2014.

[60] S. K. Garattini, D. Basile, M. Cattaneo et al., "Molecular classifications of gastric cancers: Novel insights and possible future applications," World Journal of Gastrointestinal Oncology, vol. 9, no. 5, pp. 194-208, 2017.

[61] E. Bria, S. Pilotto, M. Simbolo et al., "Comprehensive molecular portrait using next generation sequencing of resected intestinaltype gastric cancer patients dichotomized according to prognosis," Scientific Reports, vol. 6, no. 1, 2016.
[62] Y. Lin, Z. Wu, W. Guo, and J. Li, "Gene mutations in gastric cancer: a review of recent next-generation sequencing studies," Tumor Biology, vol. 36, no. 10, pp. 7385-7394, 2015.

[63] J. Cools-Lartigue, L. Baker, and L. E. Ferri, "Molecular mechanisms in gastric carcinogenesis," Gastric Cancer: Principles and Practice, pp. 35-56, 2015.

[64] M. Kanda and Y. Kodera, "Recent advances in the molecular diagnostics of gastric cancer," World Journal of Gastroenterology, vol. 21, no. 34, pp. 9838-9852, 2015. 


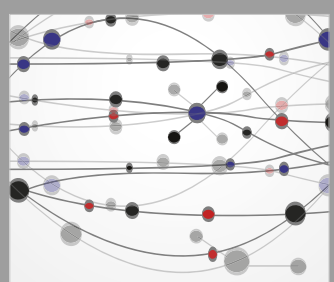

The Scientific World Journal
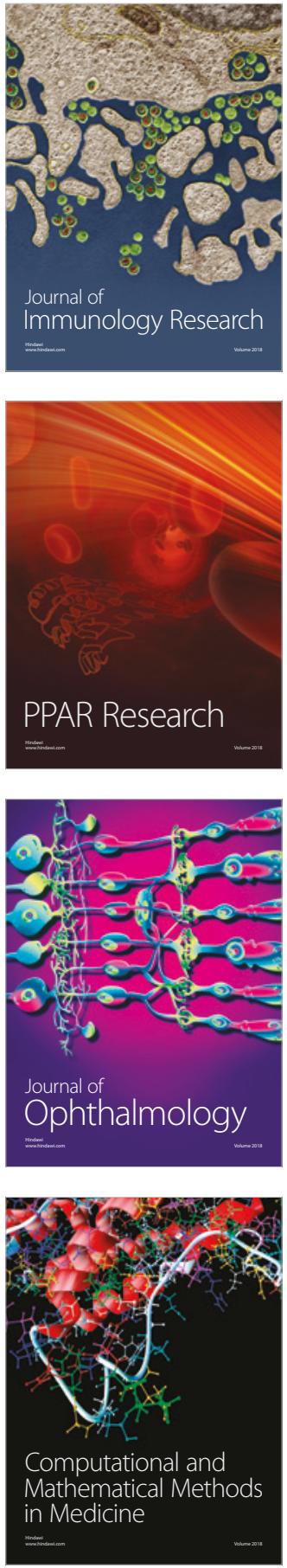

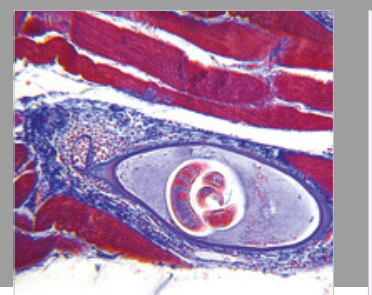

Gastroenterology Research and Practice

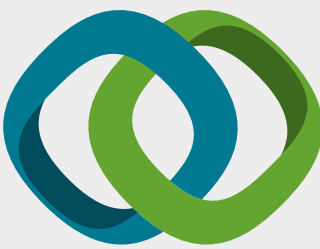

\section{Hindawi}

Submit your manuscripts at

www.hindawi.com
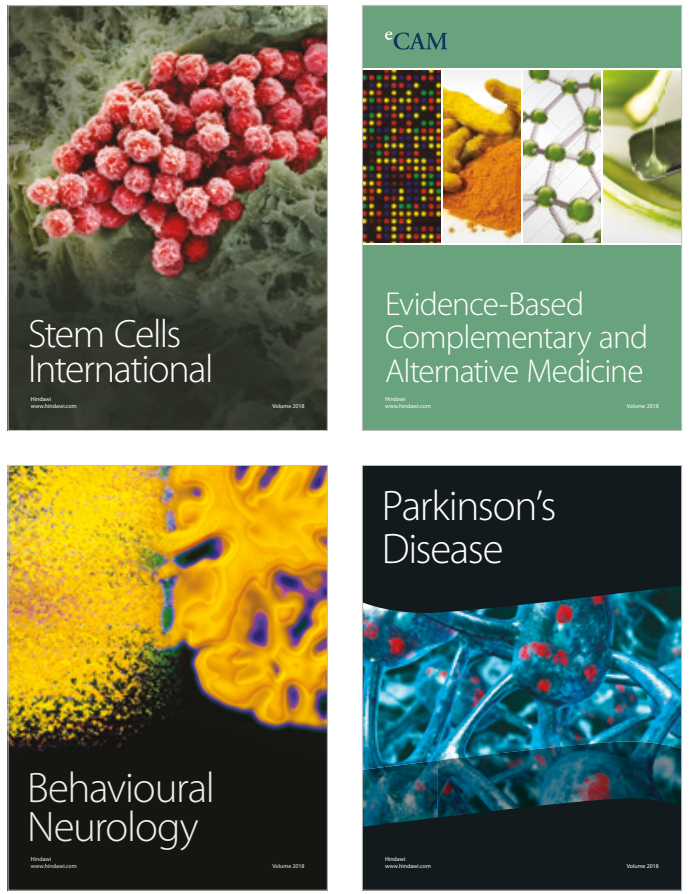

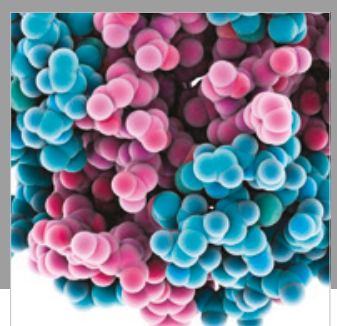

ournal of

Diabetes Research

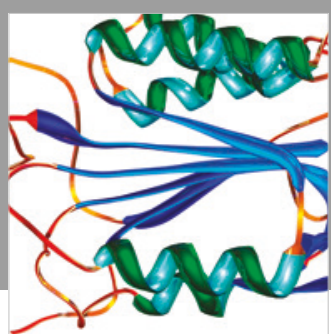

Disease Markers
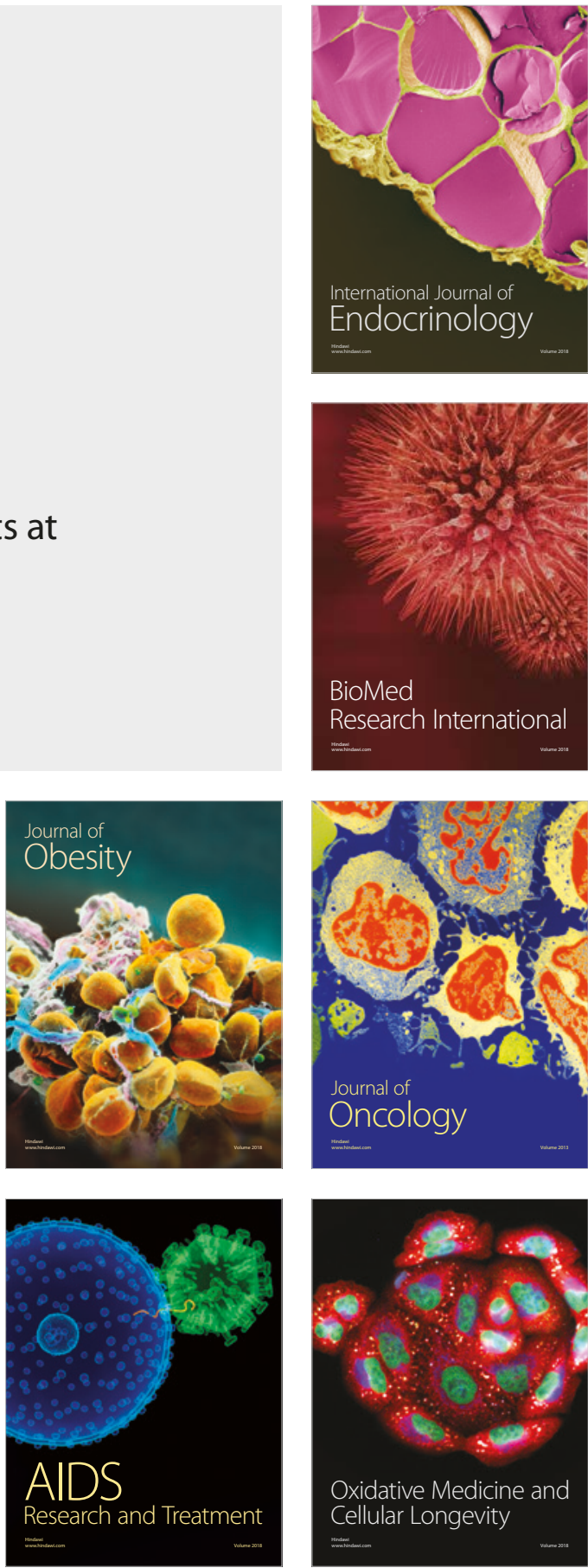